Original Article

\title{
Dysfunction of blood pressure regulation in hyperhomocyteinemia model in rats
}

\author{
Atsushi Miyajima, Masaru Bamba, Takafumi Muto and Takashi Hirota \\ Department of Biopharmaceutics, Faculty of Pharmaceutical Sciences, Tokyo University of Science \\ 2641 Yamazaki, Noda-shi, Chiba 278-8510, Japan
}

(Received October 28, 2014; Accepted January 14, 2015)

\begin{abstract}
Hyperhomocysteinemia (HHC) has been reported to be one of risk factors for cardiovascular disease. We investigated the effects of $\mathrm{HHC}$ on blood pressure regulation and its association with damage to the thoracic aorta and imbalance of redox in plasma in rats. Rats were fed a methionine enriched diet (Met diet) or a methionine and cholesterol (Met+Chol diet) enriched diet for 16 weeks to create a subchronic HHC model, in which the plasma concentration of homocysteine was about 7 times higher than that of control rats. The increase in systolic blood pressure ( $\triangle$-SBP) from sympathetic stimulation by L-epinephrine was 2- to 3-fold larger in HHC model in rats than that in control rats after several weeks of the treatment. These findings suggest that HHC deteriorates vaso-regulatory function, which could bring on an increased risk of cardiovascular events in humans. In addition, some of the elastic lamellae in the aorta were disrupted in the HHC group. However, the content of cross-linkages which give elasticity and mechanical strength in the lamellae, was not significantly different between HHC and control rats. Also plasma concentrations of thiobarbituric acid reactive substance and glutathione as indicators for redox balance in plasma were not different. In conclusion, the deterioration of vaso-regulation in HHC model in rats might be caused by the damage to elastic lamellae in the aorta, and not by oxidative stresses.
\end{abstract}

Key words: Hyperhomocysteinemia, Homocysteine, Cardiovascular event, Vaso-regulation, Elastic lamellae

\section{INTRODUCTION}

Homocysteine (Hcy), an amino acid having a thiol group, is synthesized in vivo by the demethylation of methionine. It is metabolized further with sulfur transition to cysteine through cystathionine, or returned again to methionine by methylation in the presence of 5-methylhydrofolic acid and trimethylglycine as a methyl donor. In healthy individuals, plasma concentration of Hcy is maintained constant by metabolic regulation as just described, even though that of methionine is raised by ingestion of foods.

Hyperhomocysteinemia (HHC) is well known as a genetic disease with cystathionine-synthetase deficiency, but also is a life style-related disease associated with deficiency of vitamins such as vitamin $\mathrm{B}_{6}, \mathrm{~B}_{12}$ and folic acid, and other factors such as age, smoking and alcohol abuse (Zhou et al., 2014). It was reported that patients with homocystinuria, whose plasma concentration of Hcy was markedly higher than in healthy individuals, commonly develop arteriosclerosis and thrombotic lesions early in life (McCully et al., 1969). Many researchers reported that $\mathrm{HHC}$ is an independent risk factor for cardiovascular events (Boushey et al., 1995; Refsum et al., 1998; Nygård et al., 1997). Moreover, some reports have shown that the plasma concentration of Hcy is correlated with systolic blood pressure in humans (Nygård et al., 1995; Lim and Cassano, 2002). Recently, HHC was reported to be associated with the blood pressure response to mental stress and masked hypertension (Tayama et al., 2006; Yücel et al., 2014).

Some reports have suggested the mechanism by which HHC increases the risk of cardiovascular events. For example, Charpiot et al. (1998) demonstrated that disruption of elastic lamellae in the aorta occurred in a HHC model in minipigs. Zulli et al. (1998a) reported that, in a subchronic HHC model created by feeding rats a methionine rich diet for 15 weeks, disruption of aortic elastic

Correspondence: Takashi Hirota (E-mail: hirotas5@rs.noda.tus.ac.jp) 
lamellae and thickening of the aortic wall were observed, and that such changes were further potentiated by feeding cholesterol in addition to methionine. Moreover, Raposo et al. (2004) reported that a high concentration of Hcy inhibited the activity of lysyl oxidase, which is required for the formation of cross-linkage structures in elastic lamellae, and downregulated lysyl oxidase gene expression in vascular endothelial cells. Based on these facts, it was expected that a high concentration of Hcy would disturb the elastic lamellae and reduce the cross-linkages in the aorta, and that such damage of the aorta might reduce the vaso-regulation and cause hypertension. Previously, it was reported that repeated methionine administration did not alter the blood pressure in normotensive rats (Matthias et al., 1996; Ungvari et al., 1999). However the effect of methionine-enriched diet on the ability to regulate blood pressure in response to some stimulation, such as sympathetic drugs or some stresses, in vivo still remains unclear.

On the other hand, there are some reports about endothelial dysfunction due to the oxidative stress generated from Hcy in the HHC condition. Tawakol et al. (1997) indicated that HHC is associated with impaired endothelium-dependent vasodilation in a comparative study between $\mathrm{HHC}$ and control human subjects. Lang et al. (2000) demonstrated that Hcy inhibited endothelium-dependent relaxation of rabbit aortic rings with an increase in intracellular levels of $\mathrm{O}_{2}(-)$ in the endothelial cells in an in vitro study. Tsai et al. (1994) also reported that Hcy led to an increase of smooth muscle cells in the rat aorta and a decrease of DNA synthesis in endothelial cells from human umbilical veins.

The mechanism by which HHC affects blood pressure remains unclear. Thus, in the present study, the effect of the HHC on vaso-regulation, structure of the blood vessels and redox imbalance, and the relationships among these factors were investigated using a HHC model in rats fed a methionine enriched diet.

\section{MATERIALS AND METHODS}

\section{Chemicals, reagents and diets}

Methionine, Hcy, pyridylethyl-cysteine and $\mathrm{N}$-acetylL-cysteine (NAC) were purchased from Wako Pure Chemical Industries, Ltd. (Osaka, Japan). O-phthalaldehyde and tris (2-carboxyethyl) phosphine was purchased from Tokyo Chemical Industry Co., Ltd. (Tokyo, Japan). L-Epinephrine, human angiotensin II and ethylenediaminetetraacetic acid (anhydrous) were purchased from Sigma-Aldrich (St. Louis, MO, USA). 4-Fluoro-7-sulfobenzofurazan, ammonium salt (SBD-F) was purchased from Dojindo Laboratories (Kumamoto, Japan). All other reagents and solvents in this study were commercially available and were either extra pure, guaranteed, highperformance liquid chromatography (HPLC) or liquid chromatography-mass spectrometry (LC/MS) grade.

All animal diets were purchased from Nosan Corporation (Kanagawa, Japan). Labo MR Stock ${ }^{\circledR}$ was used as a control diet and contained $0.26 \%(\mathrm{w} / \mathrm{w})$ methionine and $0.025 \%(\mathrm{w} / \mathrm{w})$ cholesterol. The composition of methionine and cholesterol in special diets was the same as those in the reports by Zulli et al. (1998a, 1998b), as follows. A methionine enriched diet (Met diet) contained $2 \%(\mathrm{w} / \mathrm{w})$ methionine; a cholesterol enriched diet (Chol diet) contained $2 \%(\mathrm{w} / \mathrm{w})$ cholesterol; and a methionine and cholesterol enriched diet (Met $+\mathrm{Chol}$ diet) contained $2 \%$ each of methionine and cholesterol. The content of the other ingredients in these special diets was the same as in the control diet.

\section{Animals}

Twenty male Sprague-Dawley rats (5 weeks old) were obtained from Japan SLC Inc. (Shizuoka, Japan) and used after 1 week of acclimatization. All rats were housed in a temperature $\left(23 \pm 1^{\circ} \mathrm{C}\right)$ and humidity $(55 \pm 5 \%)$-controlled room with 12-hr light/dark cycle. Water and diet were available ad libitum throughout the study. All experimental animals were handled in accordance with the institutional and national guidelines for the care and use of laboratory animals.

\section{Treatment of animals}

The rats were divided into 4 groups $(n=5)$ at random, and used for the study consisting of consecutive 3 periods. In the $1^{\text {st }}$ phase, the acclimatization period, all rats were fed with control diet for 1 week. Then in the $2^{\text {nd }}$ phase, the testing period, rats in each respective group were fed the Met diet, Chol diet, Met+Chol diet or control diet for 16 weeks. In the last phase, recovery period, the diet for all groups was returned to the control diet for 3 weeks.

About $0.4 \mathrm{~mL}$ of blood were collected serially from each rat with heparinized syringes under isoflurane anesthesia between 9:00 and 10:00 AM at weeks 0 (the end of the acclimatization period), 2, 4, 6, 8, 10, 12, 14 and 16 of the testing period and week 3 of the recovery period. In addition, on the first day of the recovery period, about $0.15 \mathrm{~mL}$ of blood was collected at $3,6,9,12$ and $24 \mathrm{hr}$ after the return to control diet (9:00, 12:00, $15: 00,18: 00,21: 00$ and the following day at 9:00). Plasma samples were subsequently obtained by centrifugation at $10,000 \mathrm{~g}$ and $4^{\circ} \mathrm{C}$ for $1 \mathrm{~min}$, and stored at $-80^{\circ} \mathrm{C}$ until the measurement of total Hcy, methionine, choles- 
Dysfunction of vaso-regulation in hyperhomocyteinemia

terol, triglyceride concentration, thiobarbituric acid reactive substances (TBARS) and aspartate aminotransferases (AST) activity.

Blood pressure was serially measured at week 0 (the end of the acclimatization period), at weeks $4,6,8,10$, 12,14 and 16 of the testing period, and at week 2 of the recovery period; blood collection and blood pressure measurement were done on different days. The day after the last blood pressure measurement, the thoracic aorta was isolated after the rats were euthanized by exsanguinations under isoflurane anesthesia, and prepared for histological evaluation and the determination of cross-linkages in thoracic aortas.

\section{Measurement of blood pressure}

Blood pressure with or without sympathetic stimulation was measured as described in our previous report (Miyajima et al., 2013). Briefly, systolic blood pressure (SBP) and diastolic blood pressure (DBP) were measured by a non-invasive tail-cuff/pulse transducer and a Lab Chart 7 recorder (AD Instruments Pty Ltd., Bella Vista, Australia) under pentobarbital anesthesia. Ten min before measurement of blood pressure, $10 \mu \mathrm{g} / \mathrm{kg}$ L-epinephrine was administered subcutaneously to stimulate the sympathetic nervous system. In addition, at week 2 of the recovery period the effects of angiotensin II $(0.7 \mu \mathrm{g} / \mathrm{kg})$ as another vasopressor were measured. The difference between SBP before and after administration of the vasopressor $(\Delta$-SBP) was calculated.

\section{Plasma concentrations Total Hcy, cysteine and glutathione (GSH):}

Total Hcy, which consists of oxidized forms and disulfide forms of Hcy; cysteine and glutathione were determined by HPLC after complete reduction followed by fluorescent-labeling as follows. Fifty microliters of plasma was mixed with $25 \mu \mathrm{L}$ of $1 \mathrm{mM}$ NAC (internal standard) solution and $10 \mu \mathrm{L}$ of $100 \mathrm{~g} / \mathrm{L}$ tris(2carboxyethyl)phosphine solution. The mixture was incubated at room temperature for $30 \mathrm{~min}$ to reduce disulfide bonds. After the reduction, the mixture was mixed with $90 \mu \mathrm{L}$ of $100 \mathrm{~g} / \mathrm{L}$ trichloroacetic acid and centrifuged at $13,000 \mathrm{~g}$ and $4^{\circ} \mathrm{C}$ for $10 \mathrm{~min}$ to precipitate the proteins. Then, $50 \mu \mathrm{L}$ of supernatant was mixed with $10 \mu \mathrm{L}$ of $1.55 \mathrm{M} \mathrm{NaOH}$ and $125 \mu \mathrm{L}$ of $125 \mathrm{mM}$ borate buffer (pH9.5) containing $4 \mathrm{mM}$ EDTA. In addition, the sample was mixed with $50 \mu \mathrm{L}$ of $1 \mathrm{mg} / \mathrm{mL}$ SBD-F solution and the mixture was incubated at $60^{\circ} \mathrm{C}$ for $60 \mathrm{~min}$ for the derivatization of Hcy, NAC and other thiol groups. After cooling to room temperature, the samples were used for HPLC analysis.
The derivatized Hcy, NAC, cysteine, GSH and other endogenous thiol groups were separated by a dual pump HPLC system (Shimadzu, Kyoto, Japan) using a Wakosil-5C18RS column $(4.6 \times 150$ mm, $5 \mu \mathrm{m}$; Wako Pure Chemical Industries, Ltd.) attached to a TSKgel guardgel ODS-80Ts $(3.2 \times 15 \mathrm{~mm}, 5 \mu \mathrm{m}$; Tosoh Corporation, Tokyo, Japan). The mobile phase consisted of $100 \mathrm{mM}$ acetate buffer ( $\mathrm{pH} \mathrm{4.5)}$ and methanol, and was delivered at a flow rate of $1.0 \mathrm{~mL} / \mathrm{min}$. During the measurement, the proportion of methanol in the mobile phase was increased linearly from $3 \%$ at the start to $23 \%$ at $2.0 \mathrm{~min}$, decreased to $3 \%$ at $6.0 \mathrm{~min}$, and held at this proportion until the next analysis. The fluorescence intensity was measured at $515 \mathrm{~nm}$ with excitation at $385 \mathrm{~nm}$.

\section{Methionine:}

Methionine was determined by HPLC analysis after derivatization using $O$-phthalaldehyde according to the method reported by Sun et al. (2005) with a slight modification. Briefly, $30 \mu \mathrm{L}$ of acetonitrile containing $50 \mu \mathrm{M}$ of buthylamine as an internal standard was added to $10 \mu \mathrm{L}$ of plasma and mixed well, then the sample was centrifuged at $9,000 \mathrm{~g}$ and $4^{\circ} \mathrm{C}$ for $2 \mathrm{~min}$. Ten microliters of the supernatant was mixed with $5 \mu \mathrm{L}$ of $80 \%$ methanol buffered with sodium borate $(\mathrm{pH} 10.5)$ containing $0.8 \mathrm{mg} / \mathrm{mL}$ of $O$-phthalaldehyde and $1.6 \mathrm{mg} / \mathrm{mL}$ of NAC. The mixture was incubated at room temperature for $90 \mathrm{sec}$, and then $150 \mu \mathrm{L}$ of sodium acetate buffer $(100 \mathrm{mM}$, $\mathrm{pH}$ 7.0) was added and mixed well to stop the derivatization. The derivatized samples were used for HPLC analysis.

The derivatized methionine was detected by the same HPLC system and columns as described above. The mobile phase consisted of solvent A (tetrahydrofuran $/ \mathrm{methanol} / 100 \mathrm{mM}$ acetate buffer, $\mathrm{pH} 7.0, \mathrm{v} / \mathrm{v} / \mathrm{v}=$ 5/95/900) and solvent B (methanol), and was delivered at a flow rate of $1.8 \mathrm{~mL} / \mathrm{min}$. During the measurement, the proportion of solvent B in the mobile phase was held at $20 \%$ from the start to $2.0 \mathrm{~min}$, increased linearly from $20 \%$ to $60 \%$ from 2.0 to $14.0 \mathrm{~min}$, decreased to $20 \%$ at $16.0 \mathrm{~min}$, and held at this proportion until the next analysis. The fluorescence intensity was measured at $450 \mathrm{~nm}$ with excitation at $350 \mathrm{~nm}$.

\section{Total cholesterol, triglyceride and AST:}

Total cholesterol (TC) concentration in plasma was measured by Cholesterol E-test Wako kit (Wako Pure Chemical Industries, Ltd.). Triglyceride concentration in plasma was measured by Triglyceride E-test Wako kit (Wako Pure Chemical Industries, Ltd.). AST activity in plasma was measured at weeks 0 and 16 of the testing period by Transaminase CII-test Wako kit (Wako Pure 
Chemical Industries, Ltd.). All assays were conducted using $20 \mu \mathrm{L}$ of plasma according to their instructions.

\section{TBARS:}

TBARS was measured using TBARS assay kit (Cayman Chemical Company, Ann Arbor, MI, USA) according to the instructions with slight modification. Twenty microliters of plasma was mixed with $20 \mu \mathrm{L}$ of $10 \%$ SDS solution and $800 \mu \mathrm{L}$ of $37 \mathrm{mM}$ TBA solution (containing 19.9\% acetic acid and $0.2 \mathrm{M} \mathrm{NaOH}$ ). The mixture was heated at $100^{\circ} \mathrm{C}$ for $1 \mathrm{hr}$, and then cooled on ice for $10 \mathrm{~min}$. The mixture was centrifuged at $1,600 \mathrm{~g}$ and $4^{\circ} \mathrm{C}$ for $10 \mathrm{~min}$, and then $600 \mu \mathrm{L}$ of the supernatant was taken and mixed with the same volume of 1-butanol. The mixture was centrifuged again at $1,600 \mathrm{~g}$ and $4^{\circ} \mathrm{C}$ for $15 \mathrm{~min}$. The fluorescence intensity of the supernatant was measured at $590 \mathrm{~nm}$ with excitation wave length of $540 \mathrm{~nm}$.

\section{Histological analyses of elastic lamellae}

The histological sections of aorta were prepared as described in our previous report (Miyajima et al., 2013). Briefly, fragments of thoracic aortas were fixed with the 1/2-Karnovsky' solution, and the samples were embedded in paraffin and sliced at $5 \mu \mathrm{m}$ thickness. The sections were stained with the Elastica-van Gieson methods and were observed with a light microscope $(\times 200)$.

\section{Contents of cross-linkages in thoracic aorta}

Desmosine, isodesmosine, pyridinoline and deoxypyridinoline were measured to evaluate the damage of elastin and collagen in the thoracic aorta according to our previous report (Muto et al., 2013) with slight modification. Briefly, approximately $5 \mathrm{~mm}$ long fragments of thoracic aorta were lyophilized and their dry weight was measured. $\mathrm{HCl}$ was added to the lyophilized samples, which were then heated at $110^{\circ} \mathrm{C}$ for $24 \mathrm{hr}$ and cooled at room temperature. The sample was lyophilized again and the residue was re-dissolved in mobile phase with pyridile- thyl-cysteine (an internal standard). The samples were analyzed by API-3200 LC-MS/MS system (AB Sciex, Framingham, MA, USA).

\section{Data analyses}

One-way analysis of variance with Tukey's multiple comparison tests was used to determine the statistical significance. Differences were considered statistically significant when $p<0.05$.

\section{RESULTS}

\section{Plasma concentrations of Hcy and selected endogenous substances}

To determine the Hcy exposure during the testing and recovery periods, the plasma concentrations of total Hcy were measured. In the Met and Met+Chol groups, the average plasma concentrations were $29.5 \pm 6.7$ and $39.0 \pm 7.6 \mu \mathrm{M}$, which were 7.4 and 9.8 times higher than those in the control group, respectively, in the testing period (Table 1). These results confirmed that rats in the Met and Met+Chol groups developed HHC during the treatment. In the recovery period, during the $24 \mathrm{hr}$ period after returning to the control diet the plasma concentration in the Met and Met+Chol groups quickly returned to the control level (Fig. 1).

The plasma concentrations of Hcy related metabolites were also measured. At week 2 of the testing period, the plasma concentrations of methionine, a precursor of Hcy, were about 19 times higher in the Met group and 23 times higher in the Met+Chol group compared to those in the control group (Fig. 2A, Table 1). In both groups, such significantly high concentrations were sustained in the testing period. The concentration in the Chol group was almost the same as that in the control group throughout the study. At week 3 of the recovery period the methionine levels in all groups were almost the same as in the control group. On the other hand, plasma concentrations of cysteine, which is a further metab-

Table 1. Average plasma concentration $(\mu \mathrm{M})$ of selected endogenous substances.

\begin{tabular}{lcccc}
\hline & Control & Chol & Met & Met+Chol \\
\hline Homocysteine & $4.0 \pm 0.8^{\mathrm{c}}$ & $6.0 \pm 1.0^{\mathrm{c}}$ & $29.5 \pm 6.7^{\mathrm{b}}$ & $39.0 \pm 7.6^{\mathrm{a}}$ \\
Methionine & $35 \pm 4^{\mathrm{c}}$ & $33 \pm 3^{\mathrm{c}}$ & $465 \pm 47^{\mathrm{b}}$ & $626 \pm 53^{\mathrm{a}}$ \\
Cysteine & $178 \pm 9^{\mathrm{ab}}$ & $187 \pm 7^{\mathrm{a}}$ & $163 \pm 8^{\mathrm{c}}$ & $170 \pm 9^{\mathrm{bc}}$ \\
Triglyceride & $120 \pm 8^{\mathrm{a}}$ & $137 \pm 11^{\mathrm{a}}$ & $84 \pm 14^{\mathrm{b}}$ & $97 \pm 12^{\mathrm{b}}$ \\
Total cholesterol & $67.6 \pm 9.4^{\mathrm{a}}$ & $73.8 \pm 10.2^{\mathrm{a}}$ & $67.4 \pm 18.7^{\mathrm{a}}$ & $77.1 \pm 18.5^{\mathrm{a}}$ \\
\hline
\end{tabular}

Average concentration of selected endogenous substances between the week of 2 to 16 of the testing period are shown as mean \pm S.D. $(n=5)$. Superscript letters indicate results of pairwise comparisons. Within each row, the means followed by different letters are significantly different $(p<0.05$, Tukey’s multiple comparison test). 
Dysfunction of vaso-regulation in hyperhomocyteinemia

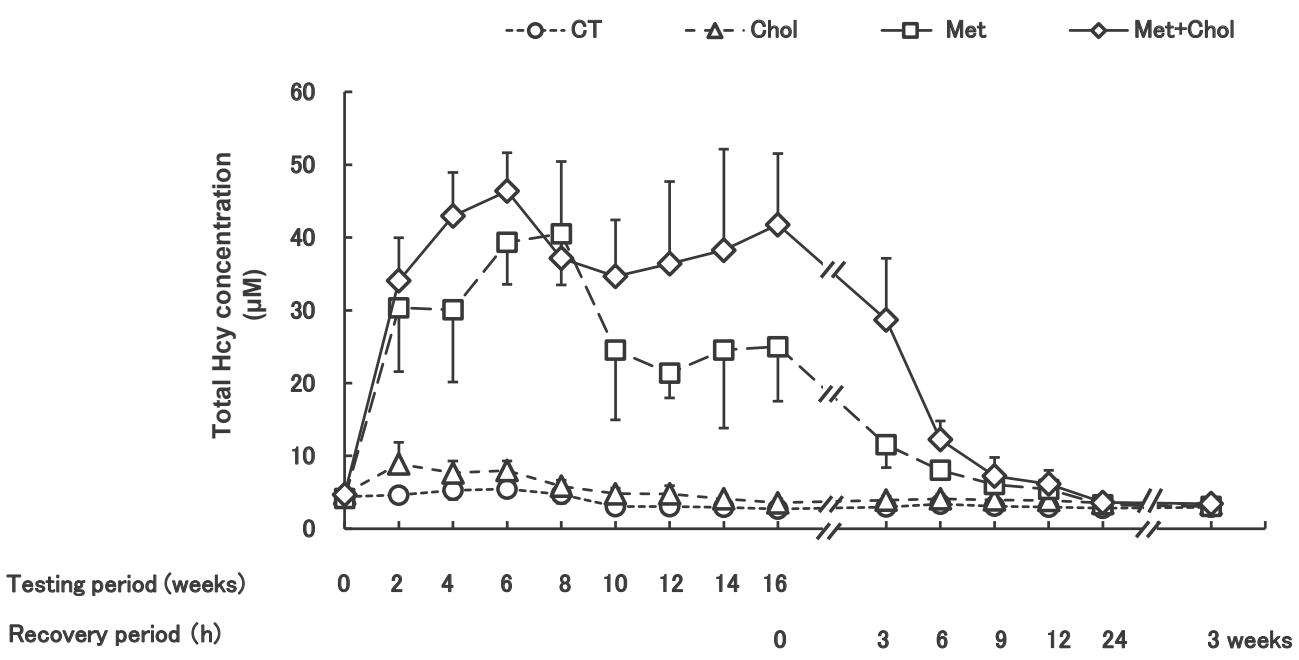

Fig. 1. Plasma concentration-time profiles of total Hcy in the testing period and the recovery period. Data are shown as mean \pm S.D. $(n=5)$.

A

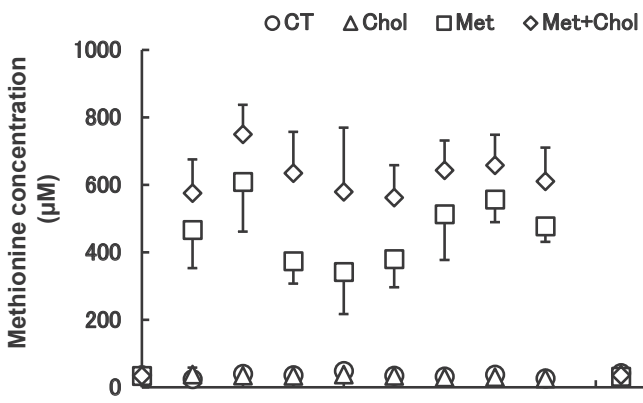

$\begin{array}{llllllllll}\text { Testing period } & 0 & 2 & 4 & 6 & 8 & 10 & 12 & 14 & 16\end{array}$

Recovery period

C

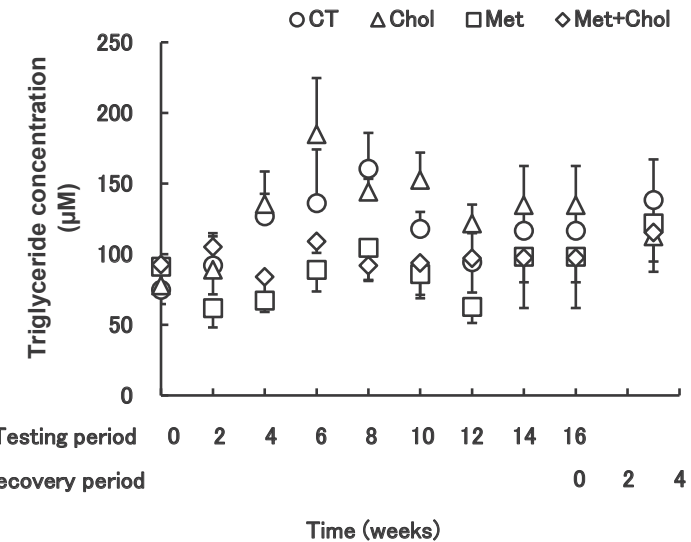

B

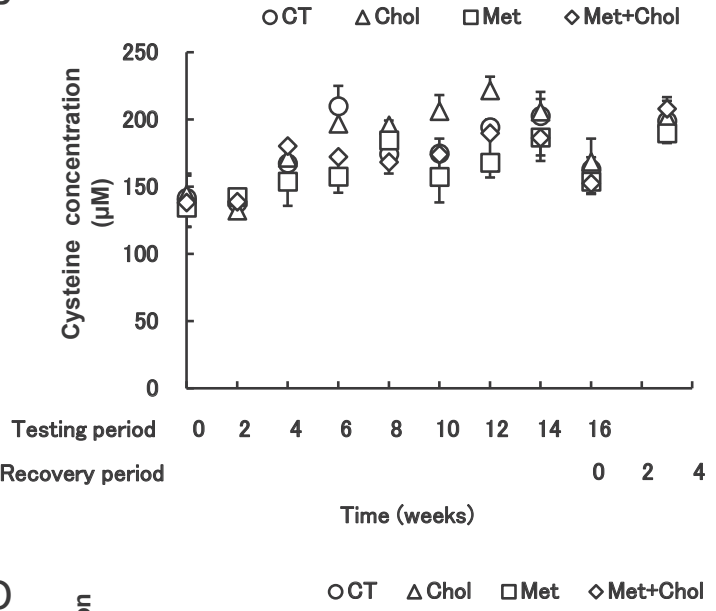

$\mathrm{D} \approx \mathrm{OCT} \Delta \mathrm{Chol}$ aMet $\diamond \mathrm{Met+Chol}$

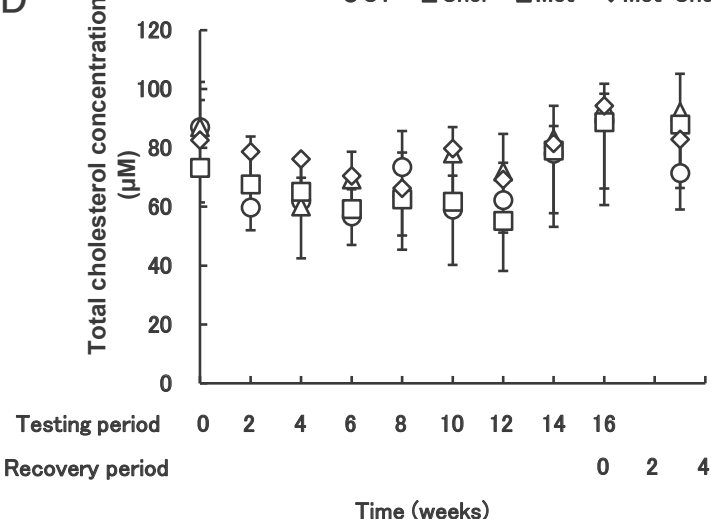

Fig. 2. Plasma concentration-time profiles of methionine (A), cysteine (B), triglyceride (C), and total cholesterol (D) in the testing and the recovery period. Data are shown as mean \pm S.D. $(n=5)$. 
A. Miyajima et al.

A

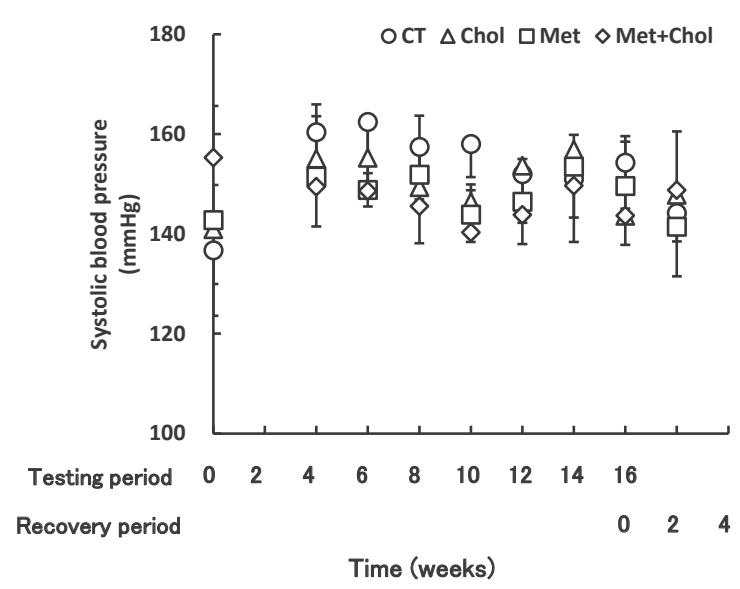

B

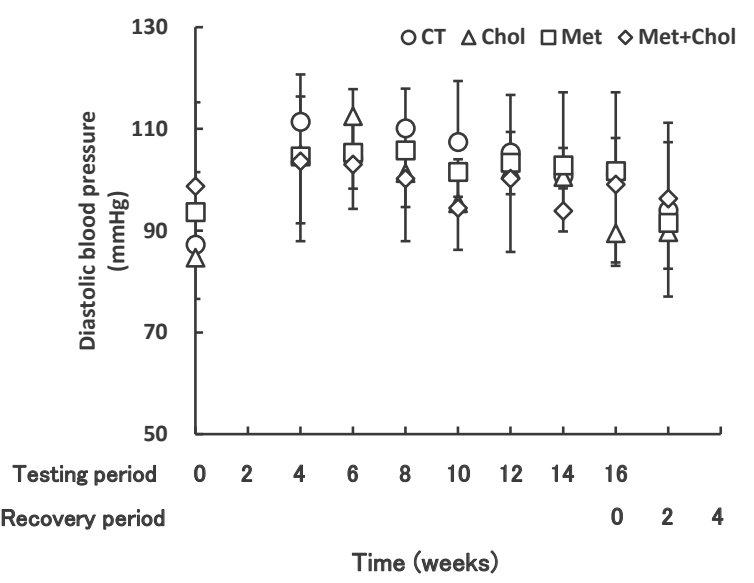

Fig. 3. SBP (A) and DBP (B) in the testing and recovery period. Data are shown as mean \pm S.D. $(n=5)$.

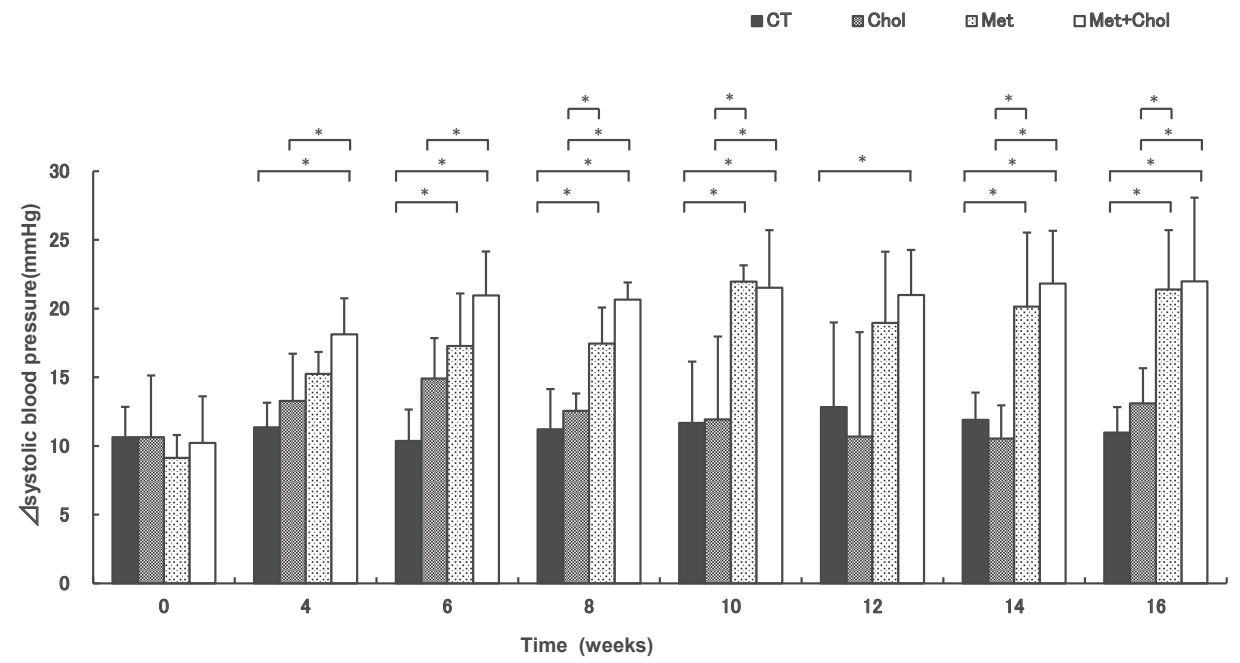

Fig. 4. Changes of blood pressure in response to L-epinephrine in the testing period. [Change in systolic blood pressure $(\Delta \mathrm{SBP})$ ] $=[$ SBP after L-epinephrine administration] $-[$ SBP before the administration]. Data are shown as mean \pm S.D. $(n=5)$. $* p<0.05$ between groups analyzed by Tukey's multiple comparison test.

olite of Hcy in the methionine metabolic pathway, did not vary greatly among all the groups throughout the study (Fig. 2B, Table 1).

The mean plasma concentration of total cholesterol was not significantly different between the control group and the other groups in the testing period (Table 1). The average plasma concentration of triglyceride was significantly lower in the Met group and the Met+Chol group compared with the control group in the testing period (Table 1). However, since the differences between the groups treated with and without methionine were relative- ly small, it was thought that they were of little physiological significance.

\section{Blood pressure and its regulation}

In the absence of vasopressor stimulation, neither SBP nor DBP was significantly different between the control group and the other groups throughout the study except at 10 weeks in the testing period (Fig. 3). The $\Delta$-SBP, the difference between SBP before and after administration of the vasopressor, was calculated as an indicator for the ability to regulate a temporary increase in blood pressure 
Dysfunction of vaso-regulation in hyperhomocyteinemia

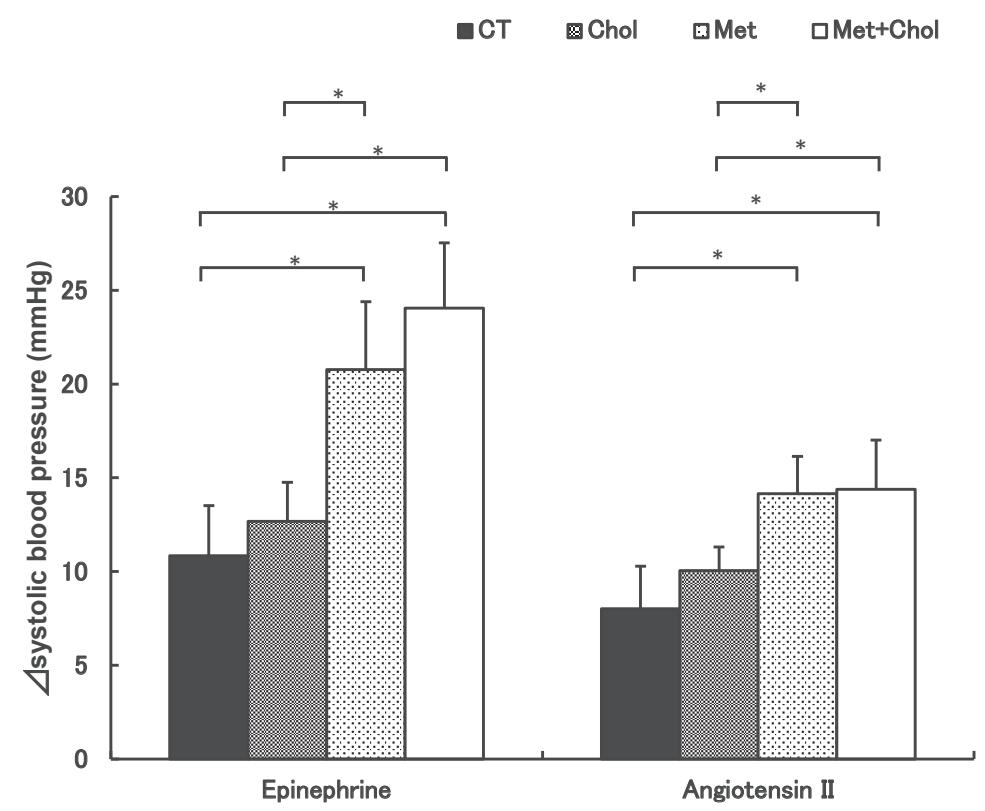

Fig. 5. Changes of blood pressure in response to L-epinephrine and angiotensin II in the recovery period. Rats were fed with Met + Chol, Met, Chol or control diet in the testing period, then their food were returned to control diet for more than 2 weeks in the recovery period. Data are shown as mean \pm S.D. $(n=5) .{ }^{*} p<0.05$ between groups analyzed by Tukey's multiple comparison test.

(Fig. 4). In the Met+Chol group at week 4 in the testing period, the $\Delta$-SBP induced by L-epinephrine was $18.1 \pm$ $2.6 \mathrm{mmHg}$, which was significantly (about 1.6-fold) larger than that in the control group $(11.4 \pm 1.8 \mathrm{mmHg})$. Also in the Met+Chol and Met groups at week 6 of the testing period, the $\Delta$-SBP were $21.0 \pm 3.2$ and $17.3 \pm 3.8 \mathrm{mmHg}$, respectively, both of which were significantly larger than that in control group $(10.4 \pm 2.3 \mathrm{mmHg})$. Until the end of the testing period, the significant enhancement of $\Delta$-SBP was observed in both the Met and Met+Chol groups compared to the control group. Furthermore, at week 2 of the recovery period, this significant enhancement of $\Delta$-SBP was still observed in the Met $+\mathrm{Chol}$ and Met groups not only when L-epinephrine was used as the vasopressor but also when angiotensin II was used (Fig. 5).

\section{Histopathological observation}

The histopathological changes of the elastic lamellae of the thoracic aorta were evaluated by optical microscopy (Fig. 6). The elastic lamellae in the aorta of control rats were clearly observed as thick bands without any branching. In the rats with HHC, some of the lamellae were disrupted and swollen. These histopathological changes in HHC were consistent with those in the previous report (Zulli et al., 1998a), although they were less severe.

\section{Contents of the cross-linkages of elastin and collagen in the aorta}

Desmosine and isodesmosine are the specific crosslinkages in elastin, and largely contribute to its elasticity (Thomas et al., 1963; Partridge et al., 1966). The contents of desmosine and isodesmosine were determined as indicators for damage to elastin by Hcy. Pyridinoline and deoxypyridinoline are the specific cross-linkages in collagen, and contribute to its mechanical strength (Eyre et al., 1984). The contents of pyridinoline and deoxypyridinoline were measured as indicators for the damage of collagen by Hcy. The contents of desmosine, isodesmosine and pyridinoline were not significantly different among the groups (Fig. 7). The deoxypyridinoline content in all groups was below the lower limit of quantification (6 ng/mL).

\section{Concentration of TBARS and GSH, and transaminase activity in the plasma}

The plasma concentrations of TBARS and GSH were measured as indicators of lipid peroxidation and antioxidant activity in plasma. Neither indicator was significantly different among the groups throughout the study (Fig. 8). The AST activity in plasma was measured as an indicator of liver function. The activity (IU/L) in 


\section{A}

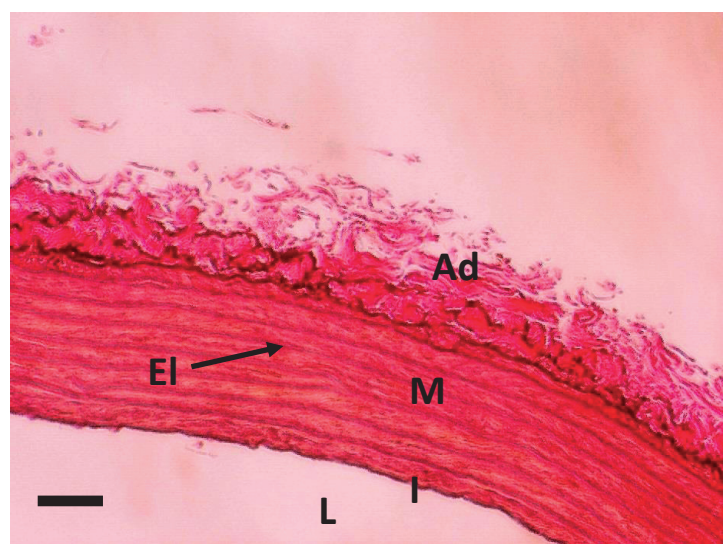

C

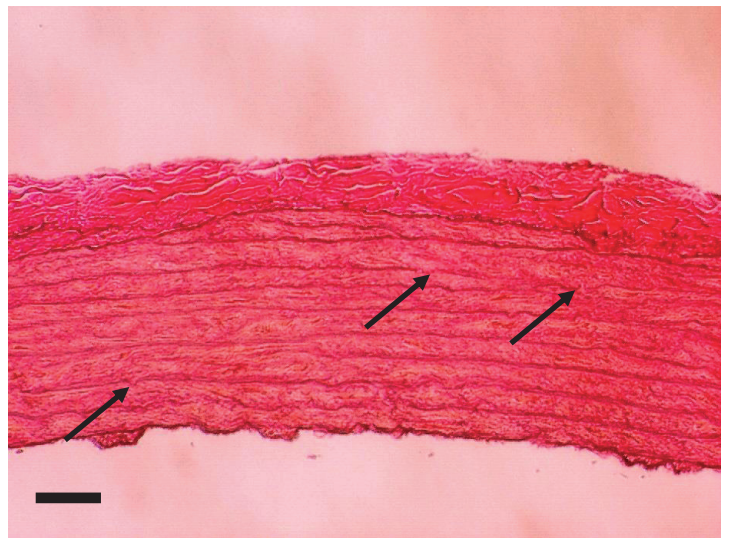

B

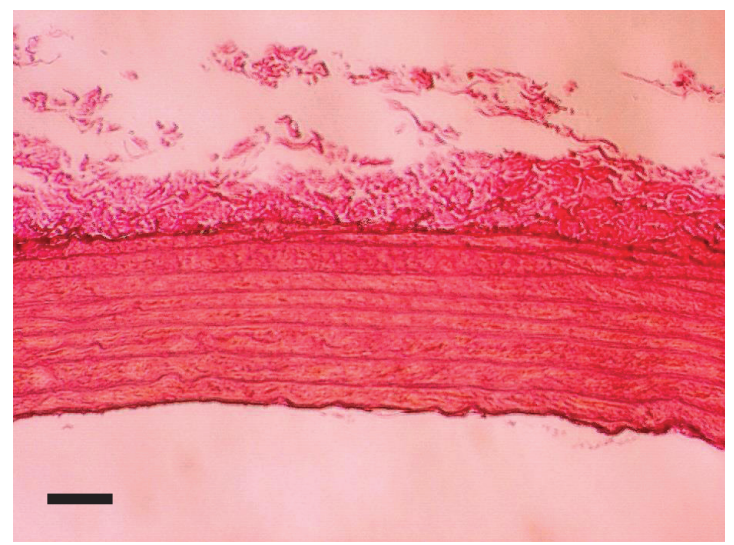

D

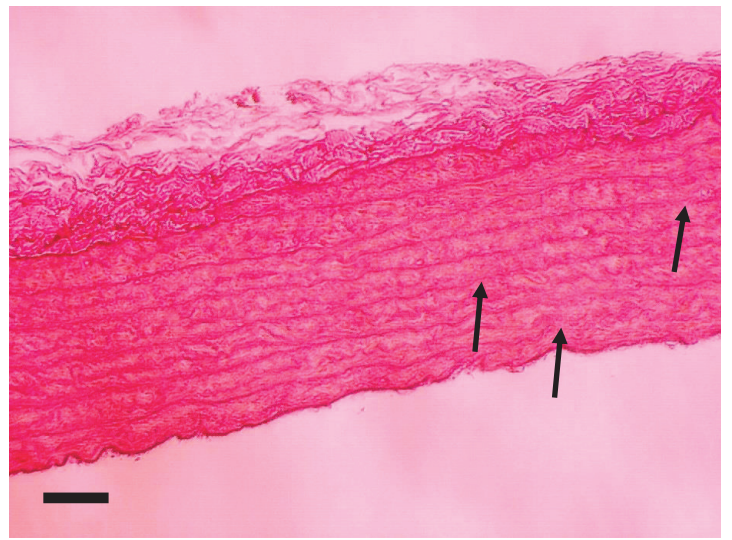

Fig. 6. Representative micrographs of elastic lamellae in the thoracic aorta of rats fed with control (A), Chol (B), Met (C), or Met+Chol (D) diet. Optical microscopy with Elastica-van Gieson method. Original magnification, $200 \times$. Bars, $40 \mu \mathrm{m}$. A) L, lumen; I, intima; M, media; Ad, adventia; El, elastic lamellae. C), D) the arrows indicate the representative parts where lamellae are disrupted and swollen.

the groups at week 16 of the testing period were as follows; Control, 7.3 \pm 0.9; Chol, 9.6 \pm 2.6 ; Met, $14.8 \pm 6.1$; Met + Chol, $8.6 \pm 2.3$. The activity of the Met group was statistically significant higher compared to the Met + Chol and control groups $(p<0.05)$. However, since the differences were relatively small, it was thought that they were of little physiological significance.

\section{DISCUSSION}

In general, the severity of human HHC is classified into 3 grades based on the plasma level of Hcy, mild (15-30 $\mu \mathrm{M})$, moderate $(30-100 \mu \mathrm{M})$ and severe (more than $100 \mu \mathrm{M})$. In the present study, since the plasma concentrations of Hcy in the Met and Met+Chol groups were in the range of $20-50 \mu \mathrm{M}$ (Fig. 1 and Table 1), our HHC model in rats could be regarded as mild or moderate HHC. Zulli et al. (1998b) reported that using older Wistar-Kyoto rats (28 weeks old at the start of treatment) the plasma concentrations of Hcy were about $85 \mu \mathrm{M}$ in Met groups and $140 \mu \mathrm{M}$ in $\mathrm{Met}+\mathrm{Chol}$ groups, values which were higher than in our study using Sprague-Dawley rats that were 6 weeks old at the start of treatment (Fig. 1, Table 1). Addition of cholesterol to the Met diet boosted the Hcy concentration in plasma by 20 to $30 \%$ compared with the Met diet in the present study, which effect was also milder than that in the report mentioned above. The milder increase of the Hcy concentration in 
Dysfunction of vaso-regulation in hyperhomocyteinemia

A

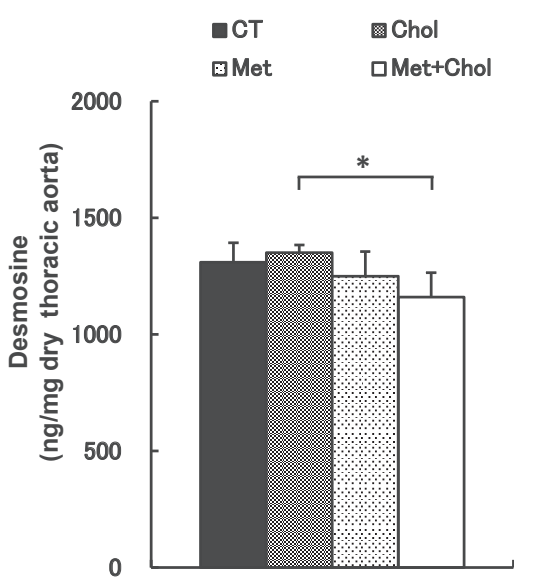

B

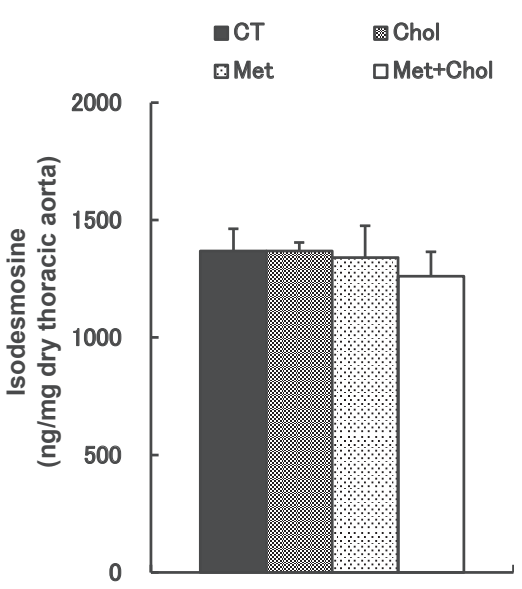

C

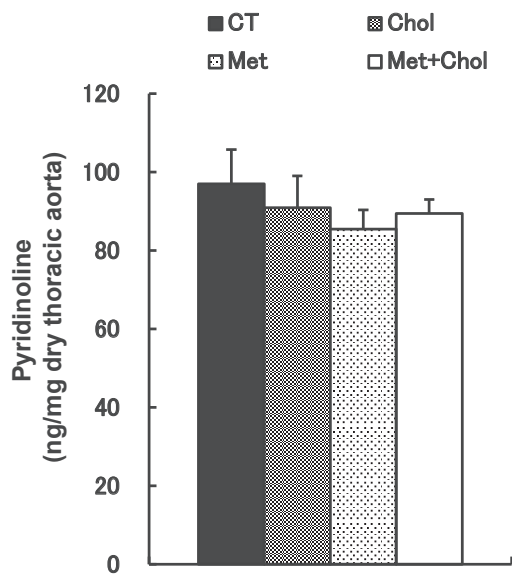

Fig. 7. Contents of desmosine (A), isodesmosine (B) and pyridinorine (C) in thoracic aorta in rats fed with Met + Chol, Met, Chol or control diets. Data are shown as mean \pm S.D. $(n=5) . * p<0.05$ between groups analyzed by Tukey's multiple comparison test.

A

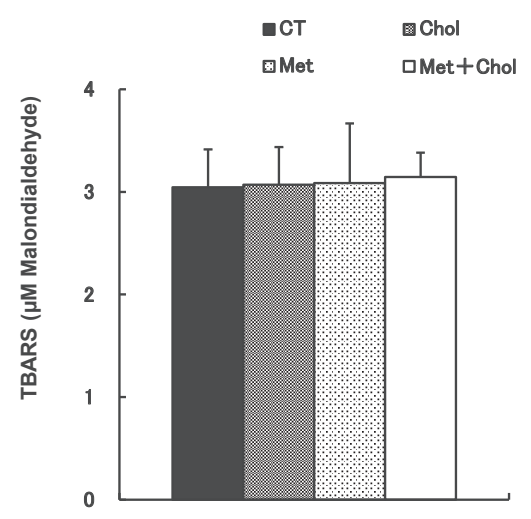

B

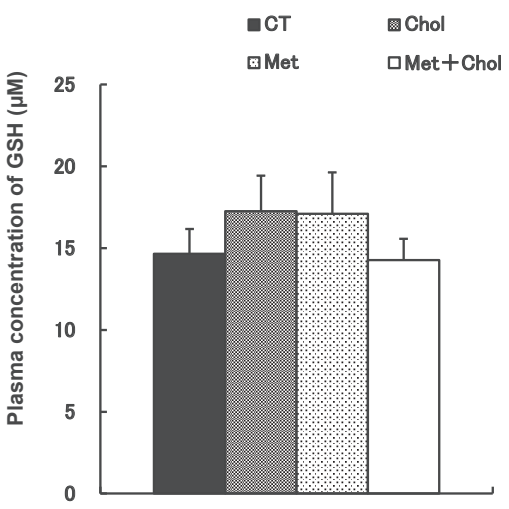

Fig. 8. Average plasma TBARS levels (A) and GSH concentration (B) during the testing period. TBARS values are expressed as equivalent malondialdehyde values. Data are shown as mean \pm S.D. $(n=5)$. ${ }^{*} p<0.05$ between groups analyzed by Tukey's multiple comparison test.

this study might be caused by the differences of the experimental conditions such as age and strain of rats. Meanwhile, the mechanism of the enhancement of increasing Hcy concentration in plasma remains unclear.

The results in this study suggested that the damage of elastic lamellae by the treatment was not serious enough to affect blood pressure in the resting condition. No differences in either SBP or DBP were observed among all the groups, regardless of the plasma Hcy concentration (Fig. 3). In general, blood pressure is mainly determined by the blood volume and the vascular caliber, and is regu- lated by the elasticity of the aorta to keep a moderate pulse pressure (i.e., the difference between SBP and DBP). With such mild impairment in the lamellae, blood pressure was well regulated by other factors, such as blood volume and/ or vascular caliber. On the other hand, the $\Delta$-SBP induced by sympathetic stimulation with L-epinephrine was significantly larger in the Met or Met+Chol group compared with the non-HHC group (Fig. 4). Thus this enhancement of $\Delta$-SBP might occur because the increase in blood pressure by L-epinephrine overwhelmed the capacity for regulation. Moreover, similar results were obtained from 
the experiments using angiotensin II instead of L-epinephrine in the rats at week 2 of the recovery period (Fig. 5). These results exhibit two very interesting features. One is that the enhancement of the $\Delta$-SBP was sustained for at least 2 weeks in the recovery period when plasma Hcy concentration had returned to normal level. This feature suggested that these harmful changes in vaso-regulation continued for more than 2 weeks after the Hcy concentration level normalized, implying the possibility of an irreversible dysfunction of vaso-regulation. The other feature is that the response occurred with vasopressors acting on different receptors. L-epinephrine and angiotensin II increase blood pressure via the constriction of blood vessels by mainly acting on the alpha 1 adrenergic receptor and angiotensin II receptor, respectively. These facts suggest the possibility that the response is not dependent on receptors of these vasopressors. Meanwhile, since in any HHC model plasma methionine level is higher than in normal animals, the possibility that the findings in this study were due to high exposure of methionine is not completely denied. We already reported that penicillamine induced disruption of elastic lamellae and dysfunction of vaso-regulation in rats (Muto et al., 2013). As one of the mechanisms for the disruption, it was thought the free SH group of penicillamine binds to extracellular matrices (Deshmukh and Nimni, 1969). Hcy, which has similar structure to penicillamine, might bind to collagen via that $\mathrm{SH}$ group. However since methionine has sulfur but not free $\mathrm{SH}$ residues in its structure, methionine is not thought to be the main factor for the harmful changes in vaso-regulation in the present study.

The elastic lamellae were partially disrupted in this rat model for HHC (Fig. 6), although the severity of disruption was clearly milder than that in the report by Zulli et al. (1998a). This difference could be explained by the differences in plasma concentration of Hcy, age and strain of rats. In addition, the cross-linkages of elastin (desmosine and isodesmosine) and collagen (pyridinoline and deoxypyridinoline) were measured as other indicators for damage to the elastin and collagen. In our previous study using rats, it was demonstrated that repeated administration of D-penicillamine (D-PA), which is a structural homologue to Hcy, disrupted elastic lamellae with a decrease of these cross-linkages and caused dysfunction of vaso-regulation (Muto et al., 2013). All of the crosslinkages in the aorta tended to be slightly decreased in the Met or Met+Chol groups compared to the control group, but were not significantly different between the treatments in this study (Fig. 7). These results indicate that such mild damage of elastic lamellae in the present study was too little to affect the content of the cross-linkages. How- ever, the possibility was not ruled out that Hcy disrupted both elastin and collagen in the same manner as penicillamine, because the contents of cross-linkages tended be to lower in the HHC model in rats (Met and Met+Chol groups) compared with the non-HHC rats (Chol and control groups). If the exposure to Hcy were higher and/or longer, the contents of cross-linkages could be decreased significantly also in this model.

Although in general Hcy induces oxidative stress, there was no significant difference of redox indicators (TBARS and GSH in plasma) among all groups (Fig. 8). Thus, at least in this study the redox environment in plasma was not affected in rats in the HHC model, indicating that oxidative stress was not a factor in the deterioration of the ability of the thoracic aorta to buffer blood pressure.

The present study demonstrated that subchronic HHC enhanced the $\Delta$-SBP after sympathomimetic stimulation, but did not affect the normal blood pressure, indicating that the capacity for vaso-regulation was reduced probably by the enhancement of the elastic lamellae disruption, and not by oxidative stress. Moreover the severity of HHC was potentiated by cholesterol intake, even though in this study it was very mild. However, in patients, considering that exposure to high concentrations of Hcy spans more than several years, the disruption of elastic lamellae and dysfunction of vaso-regulation could occur with high frequency, and accordingly increase the risk for cardiovascular events. To minimize this dysfunction of vaso-regulation, future studies are needed to develop new therapies or drugs for lowering and shortening Hcy exposure.

\section{ACKNOWLEDGMENT}

We would like to thank Dr. Donald Hinman for scientific advice and editing the manuscript.

Conflict of interest---- The authors declare that there is no conflict of interest.

\section{REFERENCES}

Boushey, C.J., Beresford, S.A., Omenn, G.S. and Motulsky, A.G. (1995): A quantitative assessment of plasma homocysteine as a risk factor for vascular disease. Probable benefits of increasing folic acid intakes. JAMA, 274, 1049-1057.

Charpiot, P., Bescond, A., Augier, T., Chareyre, C., Fraterno, M., Rolland, P.H. and Garçon, D. (1998): Hyperhomocysteinemia induces elastolysis in minipig arteries: structural consequences, arterial site specificity and effect of captopril-hydrochlorothiazide. Matrix. Biol., 17, 559-574.

Deshmukh, K. and Nimni, M.E. (1969): A defect in the intramolecular and intermolecular cross-linking of collagen caused by penicillamine. II. Functional groups involved in the interaction 
Dysfunction of vaso-regulation in hyperhomocyteinemia

process. J. Biol. Chem., 244, 1787-1795.

Eyre, D.R., Paz, M.A. and Gallop, P.M. (1984): Cross-linking in collagen and elastin. Annu. Rev. Biochem., 53, 717-748.

Lang, D.L., Kredan, M.B., Moat, S.J., Hussain, S.A., Powell, C.A., Bellamy, M.F., Powers, H.J. and Lewis, M.J. (2000): Homocysteine-induced inhibition of endothelium-dependent relaxation in rabbit aorta: role for superoxide anions. Arterioscler. Thromb. Vasc. Biol., 20, 422-427.

Lim, U. and Cassano, P.A. (2002): Homocysteine and blood pressure in the Third National Health and Nutrition Examination Survey, 1988-1994. Am. J. Epidemiol., 156, 1105-1113.

Matthias, D., Becker, C.H., Riezler, R. and Kindling, P.H. (1996): Homocysteine induced arteriosclerosis-like alterations of the aorta in normotensive and hypertensive rats following application of high doses of methionine. Atherosclerosis, 122, 201-216.

McCully, K.S. (1969): Vascular Pathology of Homocysteinemia: Implications for the Pathogenesis of Arteriosclerosis. Am. J. Pathol., 56, 111-128.

Miyajima, A., Okamoto, M., Muto, T. and Hirota, T. (2013): Disruption of elastic lamellae in aorta and dysfunction of vaso-regulation by rofecoxib in rats. J. Toxicol. Sci., 38, 719-729.

Muto, T., Miyajima, A., Bamba, M. and Hirota, T. (2013): Disruption of elastic lamellae in the aorta by D-penicillamine and its effect on vaso-regulation in rats. J. Toxicol. Sci., 38, 707-717.

Nygård, O., Nordrehaug, J.E., Refsum, H., Ueland, P.M, Farstad, M. and Vollset, S.E. (1997): Plasma homocysteine levels and mortality in patients with coronary artery disease. N. Engl. J. Med., 337, 230-236.

Nygård, O., Vollset, S.E., Refsum, H. Stensvold, I., Tverdal, A., Nordrehaug, J.E, Ueland, M. and Kvåle, G. (1995): Total plasma homocysteine and cardiovascular risk profile. The Hordaland Homocysteine Study. JAMA, 274, 1526-1533.

Partridge, S.M., Elsden, D.F., Thomas, J., Dorfman, A., Telser, A. and Ho, P.L. (1966): Incorporation of labelled lysine into the desmosine cross-bridges in elastin. Nature, 209, 399-400.

Raposo, B., Rodríguez, C., Martínez-González, J. and Badimon, L. (2004): High levels of homocysteine inhibit lysyl oxidase (LOX) and downregulate LOX expression in vascular endothelial cells. Atherosclerosis, 177, 1-8.

Refsum, H., Ueland, P.M., Nygård, O. and Vollset, S.E. (1998):
Homocysteine and cardiovascular disease. Annu. Rev. Med., 49, $31-62$.

Sun, X., Tan, Y., Yang, Z., Li, S. and Hoffman, R.M. (2005): A rapid HPLC method for the measurement of ultra-low plasma methionine concentrations applicable to methionine depletion therapy. Anticancer Res., 25, 59-62.

Tawakol, A., Omland, T., Derhard, M., Wu, J.T. and Creager, M.A. (1997): Hyperhomocyst(e)inemia is associated with impaired endothelium-dependent vasodilation in humans. Circulation, 95, 1119-1121.

Tayama, J., Munakata, M., Yoshinaga, K. and Toyota, T. (2006): Higher plasma homocysteine concentration is associated with more advanced systemic arterial stiffness and greater blood pressure response to stress in hypertensive patients. Hypertens. Res., 29, 403-409.

Thomas, J., Elsden, D.F. and Partridge, S.M. (1963): Partial structure of two major degradation products from the cross-linkages in elastin. Nature, 200, 651-652.

Tsai, J., Perrella, M.A., Yoshizumi, M., Hsieh, C., Haber, E., Schlegel, R. and Lee, M. (1994): Promotion of vascular smooth muscle cell growth by homocysteine: a link to atherosclerosis. Proc. Natl. Acad. Sci. USA, 91, 6369-6373.

Ungvari, Z., Pacher, P., Rischak, K., Szollár, L. and Koller, A. (1999): Dysfunction of nitric oxide mediation in isolated rat arterioles with methionine diet-induced hyperhomocysteinemia. Arterioscler. Thromb. Vasc. Biol., 19, 1899-1904.

Yücel, K., Bekçi, T.T., Taner, A., Kayrak, M., Korucu, E.N. and Unlü, A. (2014): Homocysteine levels in patients with masked hypertension. Anadolu. Kardiyol. Derg., 14, 357-362.

Zhou, S., Zhang, Z. and Xu, G. (2014): Notable epigenetic role of hyperhomocysteinemia in atherogenesis. Lipids Health Dis., 13, 134.

Zulli, A., Buxton, B.F., Doolan, L. and Liu, J.J. (1998a): Augmented effects of methionine and cholesterol in decreasing the elastic lamina while thickening the aortic wall in the rat aorta. Clin. Sci., 95, 589-593.

Zulli, A., Buxton, B., Doolan, L. and Liu, J.J. (1998b): Effect of homocysteine and cholesterol in raising plasma homocysteine, cholesterol and triglyceride levels. Life Sci., 62, 2191-2194. 